\title{
Sinusitis Treatment Guideline Adherence in the e-visit Setting
}

\section{A Performance Improvement Project}

Kevin L. Smith'; Dang Tran²; Bonnie L. Westra ${ }^{3}$

${ }^{1}$ Zipnosis, Minneapolis, Minnesota, United States; ${ }^{2}$ Fairview Health System, Minneapolis, Minnesota, United States; ${ }^{3}$ University of Minnesota, School of Nursing, Minneapolis, Minnesota, United States

\section{Keywords}

Clinical decision support systems, guideline adherence, quality improvement, acute bacterial rhinosinusitis (ABRS), e-visit, informatics

\section{Summary}

Background: Studies demonstrate poor guideline adherence by health care providers for the treatment of upper respiratory infections, particularly acute bacterial rhinosinusitis (ABRS), in the appropriate prescribing of antibiotic medications.

Objective: The purpose of this quality improvement project was to evaluate the effect of implementing interventions for improving adherence to a clinical practice guideline for the management of ABRS for patients treated in the e-visit setting. Interventions included: providing a report to providers of their adherence to the ABRS clinical guideline prior to the intervention, providing updated education on the ABRS guideline, and implementing a clinical decision support system reminder. Methods: A pre and post intervention evaluation design was used. Data were obtained from a retrospective electronic health record (EHR) data extract of all 316 diagnoses for ABRS in the preintervention 2-month time period and all 368 diagnoses of ABRS in the post-intervention 2-month time period. A review of the structured clinical data elements was performed to determine whether the provider adhered to the clinical guideline, meaning that only patients meeting the criteria for ABRS were to receive an antibiotic prescription.

Results and Conclusion: The interventions resulted in a 3.3\% improvement in adherence to the ABRS clinical guideline from $95.25 \%$ adherence pre-intervention to $98.4 \%$ post-intervention. These results demonstrated that the use of an educational intervention and clinical decision support resulted in improved adherence to the ABRS clinical guideline in the e-visit setting. The implications for practice could be significant in that these quality improvement interventions improve guideline adherence and reduce unnecessary prescribing of antibiotics.

Correspondence to:

Kevin L. Smith, DNP, FNP, FAANP

Zipnosis, Minneapolis

Minnesota

United States

Email: kevin@zipnosis.com
Appl Clin Inform 2016; 7: 299-307

http://dx.doi.org/10.4338/ACl-2015-10-CR-0143

received: October 20, 2015

accepted: February 15, 2016

published: May 11, 2016

Citation: Smith K, Tran D, Westra B. Sinusitis treatment guideline adherence in the e-visit setting: A performance improvement project. Appl Clin Inform 2016; 7: 299-307

http://dx.doi.org/10.4338/ACI-2015-10-CR-0143 


\section{Background}

Inappropriate antibiotic prescribing is common in spite of established upper respiratory infection (URI) guideline recommendations. Gill et al. [1] reported poor guideline adherence for the treatment of URIs, with providers prescribing antibiotics at rates in excess of the incidence of bacterial respiratory infections. Gill et al. reported $81 \%$ of patients received antibiotics for ABRS, and $65 \%$ of all URI patients with viral infections received antibiotics inappropriately. In fact, only $0.5 \%$ to $2.0 \%$ of all URI episodes result in a bacterial infection [2], which would justify using antibiotics. In Crocker et al's study, ABRS antibiotic guideline adherence rates were $43 \%$ [3].

The Centers for Disease Control and Prevention (CDC) [4] estimates that nearly $50 \%$ of antibiotics prescribed in the outpatient setting are unnecessary and that overuse of antibiotics is resulting in increased antibiotic-resistant organisms. In particular, streptococcus pneumonia, a major cause of ear and sinus infections, is resistant to one or more clinically appropriate antibiotics in $30 \%$ of severe infections.

Sinus infections prevalence rates are high. According to the National Center for Health Statistics, ABRS affects one in seven adults annually [5]. ABRS is one of the most common diagnoses made in the primary care setting, resulting in approximately 31 million individuals diagnosed each year in the United States. ABRS is the fifth most common condition diagnosed in which antibiotics are prescribed in the United States and it accounts for $15 \%$ to $21 \%$ of all antibiotics prescribed for adults in the outpatient setting [6,7]. In a study comparing e-visits (an online interaction between a health care provider and a patient) and office visits for ABRS and urinary tract infections (UTIs), Mehrotra et al. [8] demonstrated a slightly higher adherence to specific antibiotic prescribing per guideline recommendation for ABRS via e-visit (70\%) versus in-person clinic visits (67\%). However, overall antibiotic prescribing rates were slightly higher for e-visit patients for ABRS via e-visit (99\%) versus in-person clinic visits (94\%).

The diagnosis of ABRS is typically a clinical decision based primarily on the patient history. According to the Infectious Disease Society of America (IDSA) guideline [9], ABRS should be considered in patients who present with any of the following factors: (a) persistent symptoms or signs of ABRS lasting 10 or more days with no clinical improvement, (b) onset of illness with severe symptoms (fever $>102^{\circ} \mathrm{F}$ and purulent nasal discharge or facial pain) lasting at least 3 consecutive days at the beginning of illness, or (c) onset with worsening symptoms following a viral URI that lasted 5-6 days and was initially improving. The Institute for Clinical Systems Improvement (ICSI) [10] the CDC [11], and the American Academy of Otolaryngology-Head and Neck Surgery Foundation (AAO-HNS) [12] also have published evidence-based guidelines addressing appropriate diagnosis and treatment of URIs, including ABRS, which are in agreement with the IDSA guideline.

Various technology-based strategies have been studied for improving antibiotic stewardship. In a hospital based pediatric setting, Agwu et al [13] evaluated the impact of an Internet based antibiotic stewardship program which demonstrated reduction in antibiotic prescribing and cost. In a similar study using an Internet based antibiotic stewardship preapproval program over six years, Sick et al [14] demonstrated an effective reduction in the number of antibiotic doses and cost. Improved provider adherence to clinical-practice guidelines for minor acute illnesses, such as URIs, including sinusitis, has been demonstrated through the use of (CDSS) tools. These tools make it easier for the provider to choose the most appropriate treatment option and to offer feedback to providers regarding their practice patterns related to adhering to established guidelines $[15,16,17]$.

For this project, an e-visit is defined as an asynchronous encounter between a patient and a provider; there is no real-time interaction with the patient such as a phone call or real-time video encounter. The patient completes a structured online health history for a specific health problem or concern. When patients have completed entering their health history e-visit information into the software system, the provider is electronically sent a text message or pager notification that a patient e-visit is ready to be reviewed. The provider is required to log in to the secure system to review and to respond to the e-visit within one hour. The structured patient history gathering process utilizes branching logic to capture relevant patient information according to evidence based practice guidelines. The clinicians are presented with the patient history as well as guideline-based assessment and treatment options utilizing clinical decision support system (CDSS) tools. 


\section{Objectives}

The purpose of this quality improvement project was to evaluate provider adherence to ABRS clinical-practice guidelines in the e-visit setting. Conditions commonly treated via e-visits include minor acute illnesses such as upper respiratory infections, uncomplicated female bladder infections, conjunctivitis, and selected skin conditions.

\section{Methods}

\subsection{Study Design and Setting}

A pre and post intervention evaluation design was used. Each of the 29 participating e-visit providers (physicians, nurse practitioners, and physician assistants), employees of a large Midwest integrated health system, agreed to participate in the study. They received a pre-intervention report of their percentage rate of adherence to the ABRS guideline, received an educational intervention, and were provided with CDSS tools, embedded in the e-visit application, to aid in their appropriate adherence to the ABRS guideline at the point of care. Guideline adherence was measured again two months later using the ABRS guideline criteria. Comparison between the pre and post intervention adherence rates was performed by analyzing an electronic record data extract using descriptive statistics.

The patient population was limited to those seeking care via e-visits. Patients with serious medical conditions, such as congestive heart failure, and those with serious symptoms, such as severe headache pain, were triaged to receive care in a clinic or urgent care setting.

\subsection{Intervention: Guideline Adherence Improvement Strategies}

The performance-improvement project involved dissemination of current baseline provider performance for management of ABRS relative to the guideline to each of the providers (percentage adherence to the ABRS guideline in the e-visit setting), the implementation of an educational session on the ABRS guideline, and an ABRS-specific enhancement of the CDSS software functionality within the e-visit application. A description of the enhanced CDSS functionality was also included in an email communication to the providers.

The baseline review of provider ABRS guideline adherence included a two-month period, November through December 2013. A de-identified export of structured data for all ABRS e-visit encounters from the EHR for this time period was obtained. Inclusion criteria for the patient records included those who were assigned a diagnosis of ABRS, ICD 9 code 461.9, by e-visit providers. The data export was designed to include the patient symptom history, which was necessary to determine whether or not the patient met the criteria for ABRS. Key patient symptom data, derived from the structured patient interview, included separate columns for each of the symptoms. The columns were coded with basic labels: symptom duration (in days), URI symptom onset prior to facial pressure (yes/no), presence of sinus pressure (yes/no), (fever yes/no), or mild-to-moderate headache (yes/no). The structured data also included the assessment selected, by ICD 9 code, and treatment recommendations prescribed, specifically whether or not an antibiotic was prescribed, displayed in the prescription data field including the medication name, strength, dose, frequency, and duration. This report was sent to all participating providers; the performance measures included the number of ABRS e-visits performed by each of the participating providers including both individual and group aggregate measures of the percentage of cases managed according to the guidelines.

\subsection{Educational Intervention}

The ABRS educational intervention was delivered in two ways. First, the participating providers received a verbal reminder regarding the ABRS guideline at a mandatory in-person training session, which was an overall review and update of the e-visit initiative. Second, a brief email was sent to the same providers; it highlighted the key components of the ABRS guideline and described the soon- 
to-be-deployed enhancement of the CDSS, specific to the guideline. Updates to the CDSS included two changes. The structured patient history clinical note summary was modified so that the provider could see the duration (days) of symptoms text highlighted in red as CDSS for the provider. In addition, a very brief bullet-point summary of the ABRS guideline (based on ICSI, CDC, and IDSA) was added to the clinician screen, located in the visual workflow for the clinician to see while they were performing the review and clinical decision making for the e-visit patient.

\subsection{Analysis}

The structured data elements used to determine whether the clinician adhered to the guideline includes the following discrete components: duration of symptoms, fever, headache, sinus pressure, and bi-modal symptoms. The final data point is binary; it was tabulated in one of two categories as guideline adherence being met or not. The determination of adherence was based on criteria for a diagnosis of ABRS including duration of symptoms of seven days with two or more of the following symptoms: sinus pressure, worsening of pressure after initial improvement, fever, mild to moderate headache; or sinusitis symptoms for ten days or more. Figure 1 depicts the general workflow from patient interview, provider review and assessment of clinical summary, selection of diagnosis and treatment plan, and determination of adherence to the ABRS guideline.

The percentage change in guideline adherence performance from the first pre-intervention sample to the second post-intervention sample was calculated as well as statistical analysis using chisquare.

\section{Results}

The results of the data analysis prior to the educational and CDSS interventions revealed a rate of guideline adherence of $95.25 \%$. Measurement of ABRS guideline adherence post-intervention was $98.40 \%$, a $3.30 \%$, improvement in adherence to the guidelines, see $>$ Table 1 . Further analysis revealed that, in the pre-intervention group, duration of symptoms was the reason a case was not adherent to guidelines $80 \%$ of the time and $33.30 \%$ in the post-intervention group. A statistically significant change $(\mathrm{p}<.05)$ in provider adherence was found as evidenced by the $3.30 \%$ improvement from pre-intervention (15/301) to post-intervention (6/368) using chi-square analysis.

\section{Discussion}

The interventions in this project included ABRS guideline performance feedback to participating providers, an educational intervention, and enhanced CDSS focused on evidence based guideline recommendations for ABRS management. With these three interventions, there was an increase in provider adherence in the use of ABRS clinical guideline. The improvement in provider guideline adherence, as measured by the percentage change between pre- and post-intervention guideline use, was modest at $3.30 \%$. If these interventions were replicated with a larger sample size, the impact of a $3.30 \%$ improvement in ABRS guideline adherence on the estimated 31 million annual diagnoses of ABRS [6] would likely indicate a clinically significant improvement.

The data collected in this quality improvement project demonstrated that provider adherence to the ABRS may be improved with these interventions in the e-visit setting. These improvements could improve the rates of appropriate antibiotic prescribing and ultimately improve patient outcomes, such as reducing potential adverse events related to the medications and antibiotic resistance associated with overprescribing of these medications.

Since the mode of care delivery in this project was the e-visit, which is a relatively new care-delivery method, the quality improvement literature is limited in this area so comparison with other studies is limited.

One aspect of the findings that was not anticipated was that the baseline (pre-intervention) rates of guideline adherence for the treatment of ABRS for the health system were much higher than those reported in the literature. However, even though the health system baseline rate of guideline adherence was at $95.25 \%$, leaving a limited opportunity for improvement, the health system had made im- 
proving antibiotic stewardship a priority and was committed to this project to demonstrate both baseline guideline adherence and possible improvements. In addition, providing care via e-visits was relatively new for the health system, and it was committed to both monitoring and improving the quality of care in this setting.

\subsection{Limitations}

There are several limitations to this project. First, this performance improvement in ABRS guideline adherence was specific to the e-visit setting at one health system and may not apply to other e-visit settings or to the traditional ambulatory care setting, such as primary care clinics, urgent care centers, or emergency departments. Second, there were no controls in this quality improvement project to conclude that the intervention caused the improvement; rather, an improvement was observed after the interventions. Third, the use of three interventions (performance feedback, education, and CDSS) make it impossible to determine whether one, two, or all three of the interventions were needed to achieve the results of successfully improving adherence to the ABRS clinical guideline. However, a meta-analysis of sepsis guideline compliance concluded that a combination of education and process improvement results in higher compliance, so it maybe that a combination of interventions in this study had an additive effect (Damiani, 2015). Fourth, participating providers may have individually participated in an educational event or pursued self-study related to ABRS guidelines and appropriate use of antibiotics in treating URIs, thereby being influenced by factors independent of the three interventions. Finally, this study did not measure whether the most appropriate antibiotics were selected when prescribed, another important component of antibiotic stewardship. Additional study of interventions to improve adherence to antibiotic prescribing guidelines is necessary.

\section{Conclusions}

The purpose of this project was to improve adherence to ABRS treatment guidelines in the e-health visit setting. After interventions with provider using feedback, education, and CDSS interventions, an improvement of $3.30 \%$ in adherence to the ABRS guideline was observed. This project demonstrates the potential influence of these interventions on provider performance related to clinical guideline adherence. Finally, the results of this project suggests that the intervention strategies utilized could be used to improve guideline adherence for other conditions in the e-visit setting as well as treatment of similar conditions in the traditional ambulatory care setting. There is the potential to improve clinical care and ultimately improve the safety and quality of care with these strategies. Future studies are needed to further investigate the use of specific individual quality improvement interventions versus the combined interventions used here. Future studies should also focus on definitions of clinically important outcomes to determine whether the outcomes are both statistically and clinically significant.

\section{Clinical Relevance}

The goal of this project was to evaluate the effectiveness of interventions, including education and CDSS, to improve provider adherence to ABRS clinical-practice guidelines in the e-visit setting. However, The improved guideline adherence may not represent a significant clinical impact given the modest sample size and study design. Additional studies are recommended involving health systems with greater differences in baseline guideline adherence performance measures. Also, further investigation is needed to compare the differences between and the use of a single intervention versus more than one intervention for improving adherence to guidelines. 


\section{Conflict of Interest}

The authors have no conflict of interest to disclose with regards to this work. The authors report no external funding source for this project.

\section{Protection of Human and Animal Subjects}

This performance improvement project involved de-identified electronic medical record review. Human subject protection and approval was obtained through the University of Minnesota School of Nursing and the University of Minnesota Institutional Review Board (IRB) process. 


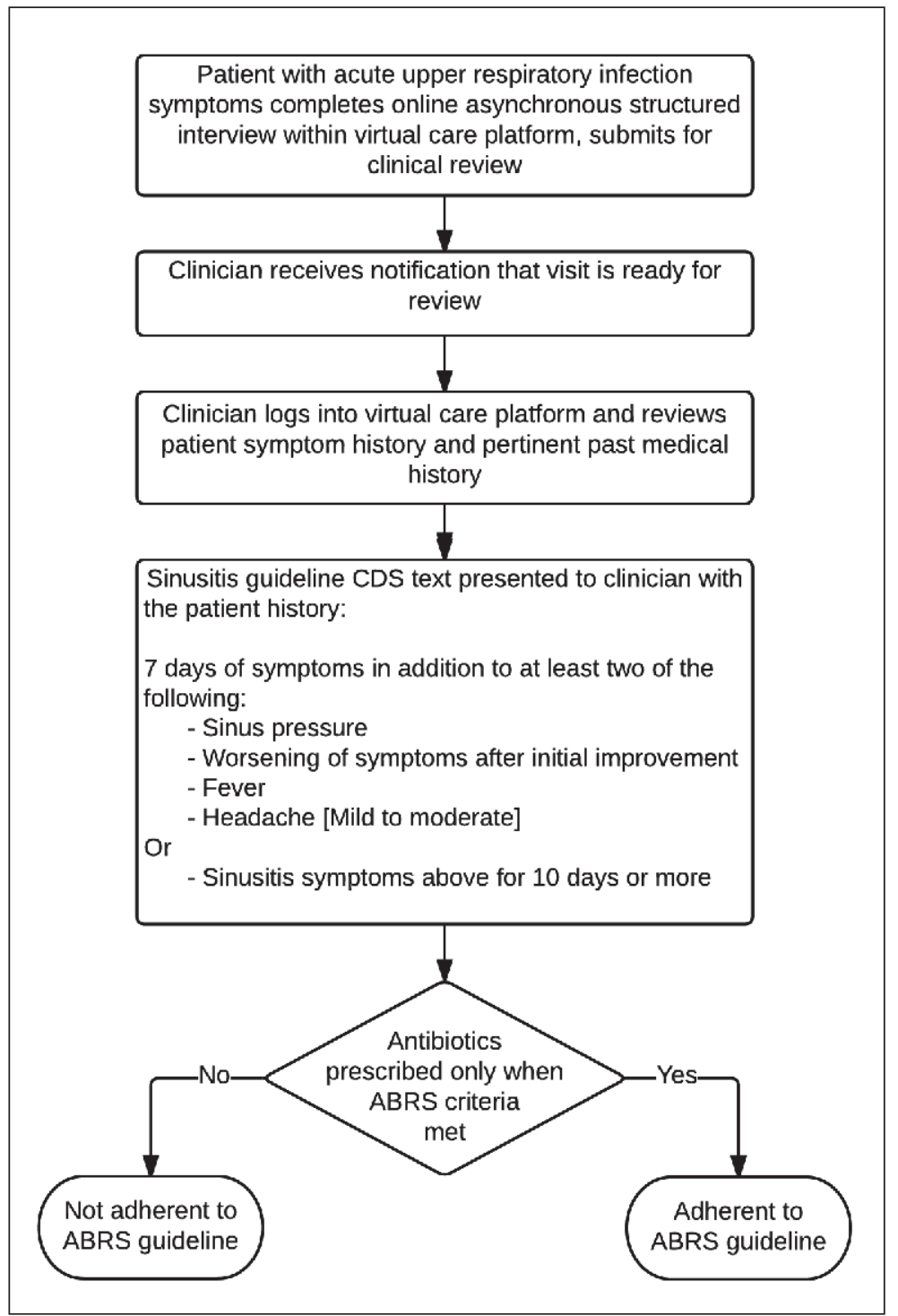

Fig. 1 Acute bacterial rhinosinusitis CDS workflow 
Table 1 Adherence to ABRS guideline before and after intervention

\begin{tabular}{|l|l|l|l|l|}
\hline Adherence to guideline & Pre intervention & Post intervention & $\%$ Change & $p$-value \\
\hline Yes & 301 & 368 & & $\leq .05$ \\
\hline No & 15 & 6 & \\
\hline Total (number) & 316 & 374 & \\
\hline Total (\%) & 95.25 & 98.40 & 3.30 \\
\hline
\end{tabular}




\section{References}

1. Gill JM, Fleischut P, Haas S, Pellini B, Crawford A, Nash DB. Use of antibiotics for adult upper respiratory infections in outpatient settings: a national ambulatory network study. Fam Med 2006; 38(5): 349-54.

2. Thomas M, Yawn BP, Price D, Lund V, Mullol J, Fokkens W. EPOS Primary Care Guidelines: European Position Paper on the Primary Care Diagnosis and Management of Rhinosinusitis and Nasal Polyps 2007 - a summary. Prim Care Respir J 2008; 17(2): 79-89.

3. Crocker A, Alweis R, Scheirer J, Schamel S, Wasser T, Levingood K. Factors affecting adherence to evidence-based guidelines in the treatment of URI, sinusitis, and pharyngitis. J Community Hosp Intern Med Perspect 2013; 3(2). Available from: http://dx.doi.org/10.3402/jchimp.v3i2.20744.

4. Centers for Disease Control and Prevention [Internet]. Antibiotic Resistance Threats in the United States, 2013. Available from: http://www.cdc.gov/drugresistance/threat-report-2013/.

5. Pleis JR, Lucas JW, Ward BW. Summary health statistics for U.S. adults: National Health Interview Survey, 2008. Vital Health Stat 10. 2009; (242): 1-157.

6. Rosenfeld RM, Andes D, Bhattacharyya N, Cheung D, Eisenberg S, Ganiats TG, Gelzer A, Hamilos D, Haydon RC 3rd, Hudgins PA, Jones S, Krouse HJ, Lee LH, Mahoney MC, Marple BF, Mitchell CJ, Nathan R, Shiffman RN, Smith TL, Witsell DL. Clinical practice guideline: adult sinusitis. Otolaryngol Head Neck Surg 2007; 137(3 Suppl): S1-31.

7. Burton MJ, Kuppersmith RB, Rosenfeld RM. Extracts from The Cochrane Library: Antibiotics for acute maxillary sinusitis. Otolaryngol Head Neck Surg 2008; 139(4): 486-9.

8. Mehrotra A, Paone S, Martich GD, Albert SM, Shevchik GJ. A comparison of care at e-visits and physician office visits for sinusitis and urinary tract infection. JAMA Intern Med 2013; 173(1): 72-4.

9. Chow AW, Benninger MS, Brook I, Brozek JL, Goldstein EJ, Hicks LA, Pankey GA, Seleznick M, Volturo G, Wald ER, File TM Jr. IDSA clinical practice guideline for acute bacterial rhinosinusitis in children and adults. Clin Infect Dis 2012; 54(8): e72-e112.

10. Snellman L, Adams W, Anderson G, Godfrey A, Gravley A, Johnson K, Marshall P, Myers C, Nesse R, Short S. Institute for Clinical Systems Improvement. Diagnosis and Treatment of Respiratory Illness in Children and Adults. Available from: http://bit.ly/RespIll. Updated January 2013.

11. Centers for Disease Control and Prevention. Acute Bacterial Rhinosinusitis: Physician Information Sheet (Adults). Available from: http://www.cdc.gov/getsmart/campaign-materials/info-sheets/adult-acute-bactrhino.html.

12. Rosenfeld RM, Piccirillo JF, Chandrasekhar SS, Brook I, Ashok Kumar K, Kramper M, Orlandi RR, Palmer JN, Patel ZM, Peters A, Walsh SA, Corrigan MD. Clinical practice guideline (update): adult sinusitis. Otolaryngol Head Neck Surg 2015 Apr; 152(2 Suppl): S1-S39.

13. Agwu AL, Lee CK, Jain SK, Murray KL, Topolski J, Miller RE, Townsend T, Lehmann CU. A World Wide Web-based antimicrobial stewardship program improves efficiency, communication, and user satisfaction and reduces cost in a tertiary care pediatric medical center. Clin Infect Dis 2008 Sep 15; 47(6): 747-53.

14. Sick AC, Lehmann CU, Tamma PD, Lee CK, Agwu AL. Sustained savings from a longitudinal cost analysis of an internet-based preapproval antimicrobial stewardship program. Infect Control Hosp Epidemiol 2013 Jun; 34(6): 573-80.

15. Woodburn JD, Smith KL, Nelson GD. Quality of care in the retail health care setting using national clinical guidelines for acute pharyngitis. Am J Med Qual 2007; 22(6): 457-62.

16. Alweis R, Greco M, Wasser T, Wenderoth S. An initiative to improve adherence to evidence-based guidelines in the treatment of URIs, sinusitis, and pharyngitis. J Community Hosp Intern Med Perspect 2014; 4(1). Available from: http://dx.doi.org/10.3402/jchimp.v4.22958.

17. Castillo RS, Kelemen A. Considerations for a successful clinical decision support system. Comput Inform Nurs 2013; 31(7): 319-26.

18. Damiani E, Donati A, Serafini G, Rinaldi L, Adrario E, Pelaia P, Busani S, Girardis M. Effect of performance improvement programs on compliance with sepsis bundles and mortality: a systematic review and meta-analysis of observational studies. PLoS One 2015; 10(5): e0125827. 